\title{
MIGUEL MOTAS GUZMÁN: UBICACIÓN EN CIENCIAS BIOMÉDICAS DE LA DOCENCIA EN DEONTOLOGÍA, MEDICINA LEGAL Y LEGISLACIÓN SANITARIA ${ }^{1}$
}

\section{PLACEMENT OF DEONTOLOGY, LEGAL MEDICINE AND SANITARY LEGISLATION TEACHING IN BIOMEDICAL SCIENCES}

RESUMEN: La docencia en deontología, medicina legal y legislación sanitaria, no puede desarrollarse con unos mínimos de calidad si no está ubicada en los últimos años de grado o licenciatura. Sus especiales connotaciones le hacen precisar de unos conocimientos previos imprescindibles para su correcto entendimiento. A su vez su importancia y repercusión en el código deontológico de cada profesional, sugieren encarecidamente su ubicación en últimos cursos. Se abordan los perjuicios derivados de la ubicación de esta materia en cursos iniciales de ciencias biomédicas, por una interpretación forzada y sesgada del libro blanco de las titulaciones, por encima de criterios de calidad docente.

ABSTRACT: Deontology, legal medicine and sanitary legislation teaching, will not achieve some minimum quality levels unless it is taught within the last courses of a degree. The distinctive features of these subjects make it necessary to have some previous knowledge essential for their correct understanding. Equally, their importance and repercussion in the code of ethics of each professional, strongly suggests its location in last courses. We address the damages derived from the location of these subjects in initial courses in biomedical sciences, caused by a forced and biased interpretation of the white paper of the degrees, above any other teaching quality criteria.

PALABRAS CLAVE: ubicación, deontología, medicina legal y legislación sanitaria, ciencias biomédicas.

KEYWORDS: placement, deontology, legal medicine and sanitary legislation, biomedical sciences.

Los estudios de Veterinaria están regulados por la Directiva Europea 36/2005 (http://www.unizar.es/eees/doc/directiva200536ec.pdf, página 224) parcialmente modificada por la 55/2013 (la modificación no afecta a la estructura de los estudios), y traspuesta a la legislación española por el Decreto $1837 / 2008$.

\footnotetext{
${ }^{1}$ Profesor toxicología, deontología, medicina legal y legislación veterinaria. Deseo expresar mi agradecimientos al profesor compañero y amigo Dr. D. Antonio Rouco Yáñez, Profesor de economía agraria, antiguo Decano de la Facultad de Veterinaria de Murcia y expresidente de la Conferencia de decanos de Veterinaria, por su valiosa e inestimable ayuda.
} 
La Directiva en cuestión establece: el programa de estudios necesarios para obtener los títulos de formación de veterinario incluirá, por lo menos, las materias enumeradas a continuación. La enseñanza de una o de varias de estas materias podrá impartirse en el marco de las otras asignaturas o en conexión con ellas.

A. Materias básicas

- Física.

- Química.

- Zoología.

- Botánica.

- Matemáticas aplicadas a las ciencias biológicas.

B. Materias específicas:

a. Ciencias básicas:

- Anatomía (incluidas histología y embriología).

- Fisiología.

- Bioquímica.

- Genética.

- Farmacología.

- Farmacia.

- Toxicología.

- Microbiología.

- Inmunología.

- Epidemiología.

- Deontología.

b. Ciencias clínicas:

- Obstetricia.

- Patología (incluida la anatomía patológica).

- Parasitología.

- Medicina y cirugía clínicas (incluida la anestesiología).

- Clínica de los animales domésticos, aves de corral y otras especies animales.

- Medicina preventiva.

- Radiología.

- Reproducción y trastornos de la reproducción.

- Policía sanitaria.

- Medicina legal y legislación veterinarias.

- Terapéutica.

- Propedéutica.

c. Producción animal:

- Producción animal.

- Nutrición.

- Agronomía.

- Economía rural.

- Crianza y salud de los animales. 
- Higiene veterinaria.

- Etología y protección animal.

d. Higiene alimentaria:

- Inspección y control de los productos alimenticios animales o de origen animal.

- Higiene y tecnología alimentarias.

- Prácticas (incluidas las prácticas en mataderos y lugares de tratamiento de los productos alimenticios).

La formación práctica podrá realizarse en forma de período de trabajo en prácticas, siempre que este sea con dedicación exclusiva bajo el control directo de la autoridad u organismo competentes y no exceda de seis meses dentro de un período global de formación de cinco años de estudios.

La distribución de la enseñanza teórica y práctica entre los distintos grupos de materias deberá ponderarse y coordinarse de tal manera que los conocimientos y la experiencia se puedan adquirir de forma que el veterinario pueda desempeñar todas las tareas que le son propias.

Se ha transcrito entero el texto de la DE referente al Veterinario porque con el "Plan Bolonia" o EEES sigue siendo de obligado cumplimiento, razón por la que tenemos un Grado de 5 años y no de 4 como en la mayoría de títulos.

Otra cuestión a tener en cuenta en la Directiva es la necesidad de atender a todas las materias citadas, pudiendo haber una estructura de materias o asignaturas diferente a la que aparece en dicha Directiva, mediante la unión o desdoblado de materias, como se realiza en casi toda Europa.

Con la llegada del Plan Bolonia en vez de en asignaturas, se centra la atención en COMPETENCIAS, que después deberán trasladarse a asignaturas. En este sentido, ANECA en 2004 encarga a las Conferencias de Decanos que elaboren los denominados Libros Blancos de los Grados (Libro Blanco Veterinaria: http://www.aneca.es/var/media/150400/libroblanco jun05 veterinaria.pdf). En dichos libros los estudios se estructuran en Módulos que se corresponden con competencias, que más tarde se trasladarán a asignaturas.

\begin{tabular}{|c|c|}
\hline Módulos Libro Blanco & Competencias \\
\hline $\begin{array}{l}\text { Ciencias Básicas (propuesta } \\
\text { de asignación de } 6,75 \text { a } 11,25 \\
\text { ECTS) }\end{array}$ & $\begin{array}{l}\text { Principios básicos de la biometría y } \\
\text { estadística aplicados a las ciencias } \\
\text { veterinarias. Bases físicas y químicas de los } \\
\text { procesos biológicos y sus aplicaciones a las } \\
\text { ciencias veterinarias. Morfología, bionomía y } \\
\text { sistemática de los animales y vegetales de }\end{array}$ \\
\hline
\end{tabular}




\begin{tabular}{|c|c|}
\hline & interés veterinario. \\
\hline $\begin{array}{l}\text { Estructura y Función } \\
\text { (propuesta de asignación de } \\
47,25 \text { a } 51,75 \text { ECTS) }\end{array}$ & $\begin{array}{l}\text { Estructura de la célula eucariota y su } \\
\text { organización en tejidos y órganos. } \\
\text { Morfología, topografía y estructura de los } \\
\text { órganos y sistemas. Excitabilidad y } \\
\text { comunicación celular. Funcionamiento y } \\
\text { regulación de los aparatos y sistemas } \\
\text { corporales. Homeostasis. Desarrollo } \\
\text { ontogénico, anomalías congénitas y } \\
\text { aplicaciones de la embriología. Bases } \\
\text { moleculares y genéticas de los procesos } \\
\text { biológicos. } \\
\text { Principios básicos de la biotecnología } \\
\text { genética y de la genética de poblaciones. } \\
\text { Características etnológicas y productivas, con } \\
\text { especial referencia al manejo. Bases del } \\
\text { comportamiento animal y del proceso de } \\
\text { domesticación }\end{array}$ \\
\hline \begin{tabular}{llr|} 
Agentes biológicos & y \\
alteraciones de la Estructura y \\
Función (propuesta r de \\
asignación de 24,75 a 29,25 \\
ECTS)
\end{tabular} & $\begin{array}{l}\text { Estudio de los microorganismos y parásitos } \\
\text { que afectan a los animales y de aquellos que } \\
\text { tengan una aplicación industrial, } \\
\text { biotecnológica o ecológica. Bases y } \\
\text { aplicaciones técnicas de la respuesta } \\
\text { inmune. Nosología. Descripción y patogenia } \\
\text { de las alteraciones generales de la estructura } \\
\text { y función de las células, tejidos, órganos y } \\
\text { sistemas. }\end{array}$ \\
\hline $\begin{array}{l}\text {, Ética y Legislación } \\
\text { sta de asignación de } \\
3,50 \text { ECTS) }\end{array}$ & $\begin{array}{l}\text { Principios éticos de la profesión } \\
\text { veterinaria. Normativa y reglamentación } \\
\text { veterinaria. Bienestar y protección animal. } \\
\text { Bioética. Marketing y gestión empresarial de } \\
\text { ámbito general y veterinario. }\end{array}$ \\
\hline $\begin{array}{l}\text { Fundamentos del Diagnóstico } \\
\text { y la Terapéutica (propuesta de } \\
\text { asignación de } 21,37 \text { a } 25,87 \\
\text { ECTS) }\end{array}$ & $\begin{array}{l}\text { Métodos y procedimientos de exploración } \\
\text { clínica, técnicas } \\
\text { complementarias y su iagnósticas } \\
\text { Diagnóstico por imagen y radiobioción. } \\
\text { Necropsia. Reconocimiento y diagnóstico de } \\
\text { los distintos tipos de lesiones y su asociación } \\
\text { con los procesos patológicos. Bases } \\
\text { farmacológicas generales y estudio de los } \\
\text { distintos tipos de drogas. }\end{array}$ \\
\hline $\begin{array}{l}\text { Ciencias Clínicas (propuesta } \\
\text { de asignación de } 31,50 \text { a } \\
36,00 \text { ECTS) }\end{array}$ & $\begin{array}{l}\text { Estudio clínico del individuo enfermo y los } \\
\text { tratamientos médicos, quirúrgicos o higiénico- } \\
\text { dietéticos que requiera, así como de las } \\
\text { enfermedades esporádicas que afecten a } \\
\text { colectivos. Diagnóstico. }\end{array}$ \\
\hline
\end{tabular}




\begin{tabular}{|c|c|}
\hline & $\begin{array}{l}\text { Técnicas quirúrgicas utilizadas en veterinaria. } \\
\text { Anestesia y reanimación animal. } \\
\text { Reproducción, parto y puerperio: cuidados y } \\
\text { enfermedades. Reproducción asistida. } \\
\text { Farmacoterapia. Identificación y estudio de } \\
\text { los tóxicos naturales y de síntesis. } \\
\text { Toxicología animal y medioambiental. }\end{array}$ \\
\hline $\begin{array}{l}\text { Sanidad Animal (propuesta de } \\
\text { asignación de 23,62 a } 28,12 \\
\text { ECTS) }\end{array}$ & $\begin{array}{l}\text { Transmisión y mantenimiento de las } \\
\text { enfermedades y métodos de estudio de las } \\
\text { enfermedades en las poblaciones. } \\
\text { Enfermedades infecciosas y parasitarias de } \\
\text { interés veterinario incluyendo su diagnóstico } \\
\text { y lucha. Zoonosis y Salud Pública. Promoción } \\
\text { de la salud en los colectivos animales con el } \\
\text { fin de obtener el máximo rendimiento } \\
\text { económico de una forma social, ética y } \\
\text { sanitariamente aceptables. Medidas técnicas } \\
\text { y reglamentos para la prevención, control y } \\
\text { erradicación de las enfermedades animales. }\end{array}$ \\
\hline $\begin{array}{l}\text { Producciones Animales } \\
\text { (propuesta de asignación de } \\
28,12 \text { a } 32,62 \text { ECTS) }\end{array}$ & $\begin{array}{l}\text { Bases de la producción animal: sistemas } \\
\text { tradicionales y actuales. Materias primas para } \\
\text { la alimentación animal: características, } \\
\text { producción y conservación. Bases de la } \\
\text { nutrición animal, formulación de raciones y } \\
\text { fabricación de piensos. Aplicaciones } \\
\text { genéticas a programas de mejora y salud. } \\
\text { Estrategias y procedimientos reproductivos } \\
\text { aplicados a la producción. Fundamentos de } \\
\text { instalaciones ganaderas e higiene ambiental. } \\
\text { Economía del proceso productivo y } \\
\text { comercialización. Desarrollo sostenible }\end{array}$ \\
\hline 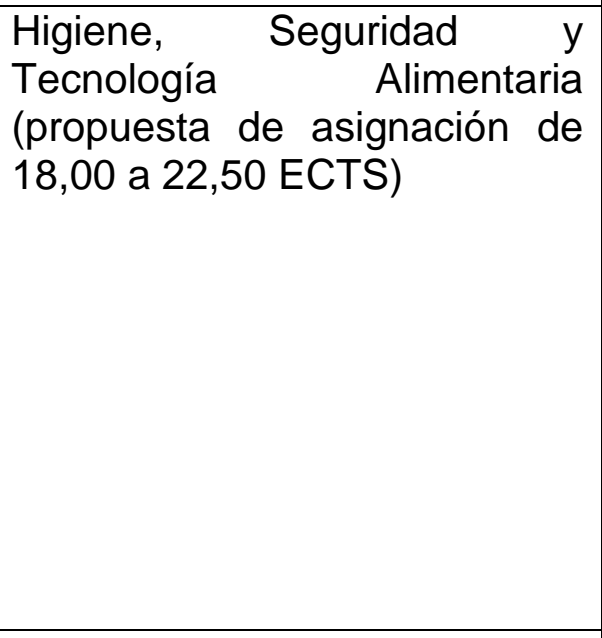 & $\begin{array}{l}\text { Componentes y características de los } \\
\text { alimentos. Procesos tecnológicos de } \\
\text { obtención, conservación y transformación de } \\
\text { los alimentos. Cambios, alteraciones y } \\
\text { adulteraciones que pueden sufrir. } \\
\text { Criterios sanitarios y bases legales de la } \\
\text { inspección. Inspección veterinaria ante y } \\
\text { post-mortem. } \\
\text { Inspección de establecimientos y productos. } \\
\text { Buenas prácticas higiénicas, análisis de } \\
\text { peligros y puntos de control críticos. Control } \\
\text { de manipulación y tratamientos. Seguridad } \\
\text { Alimentaria y Salud Pública }\end{array}$ \\
\hline $\begin{array}{lr}\text { Prácticas } & \text { Tuteladas } \\
\text { (propuesta de } & \text { asignación de }\end{array}$ & $\begin{array}{l}\text { Prácticas preprofesionales en hospi } \\
\text { clínicos veterinarios, granjas, plantas } p\end{array}$ \\
\hline
\end{tabular}




\section{0,00 ECTS)}

departamentos con docencia en la
licenciatura de veterinaria así como estancias
en mataderos, empresas y organismos
externos de ámbito veterinario o afín.

Una de las conclusiones del Libro Blanco del Grado en Veterinaria, fue que para desarrollar todo el currículo eran necesarios 330 ECTS (5,5 cursos).

Al mismo tiempo, al ser Veterinaria una carrera profesionalizante así como una profesión regulada, el Gobierno para normativizarla (al igual que con otras carreras de las mismas características) emite una Orden Ministerial, de obligado cumplimiento: Orden ECI 333/2008 (http://www.boe.es/boe/dias/2008/02/15/pdfs/A08355-08357.pdf), donde se mantiene la estructura del Libro Blanco de Módulos ligados a competencias, pero con ligeras variaciones:

1. Refunde los módulos, denominándolos igual que lo hace la Directiva Europea.

2. Respeta las competencias del Libro Blanco.

El resultado fue el siguiente:

\section{Módulos Libro Blanco}

De formación básica común (83 ECTS mínimo)

\section{Competencias}

Biometría y estadística aplicados a las ciencias veterinarias. Bases físicas y químicas de los procesos biológicos y sus aplicaciones a las ciencias veterinarias. Morfología, bionomía y sistemática de los animales y vegetales de interés veterinario.

Estructura de la célula eucariota y su organización en tejidos y órganos. Morfología, topografía y estructura de los órganos y sistemas. Excitabilidad y comunicación celular.

Funcionamiento y regulación de los aparatos y sistemas corporales. Homeostasis. Desarrollo ontogénico, anomalías congénitas y aplicaciones de la embriología. Bases moleculares y genéticas de los procesos biológicos. Principios básicos de la biotecnología genética y de la genética de poblaciones. Características etnológicas y productivas, con especial referencia al manejo. Bases del comportamiento animal y del proceso de domesticación.

Estudio de los microorganismos y parásitos que afectan a los animales y de aquellos que 


\begin{tabular}{|c|c|}
\hline & $\begin{array}{l}\text { tengan una aplicación industrial, } \\
\text { biotecnológica o ecológica. Bases y } \\
\text { aplicaciones técnicas de la respuesta } \\
\text { inmune. Nosología. Descripción y patogenia } \\
\text { de las alteraciones generales de la estructura } \\
\text { y función de las células, tejidos, órganos y } \\
\text { sistemas. } \\
\text { Principios éticos de la profesión } \\
\text { veterinaria. Normativa y reglamentación } \\
\text { veterinaria. Bienestar y protección animal. } \\
\text { Bioética. Marketing y gestión empresarial de } \\
\text { ámbito general y veterinario. }\end{array}$ \\
\hline 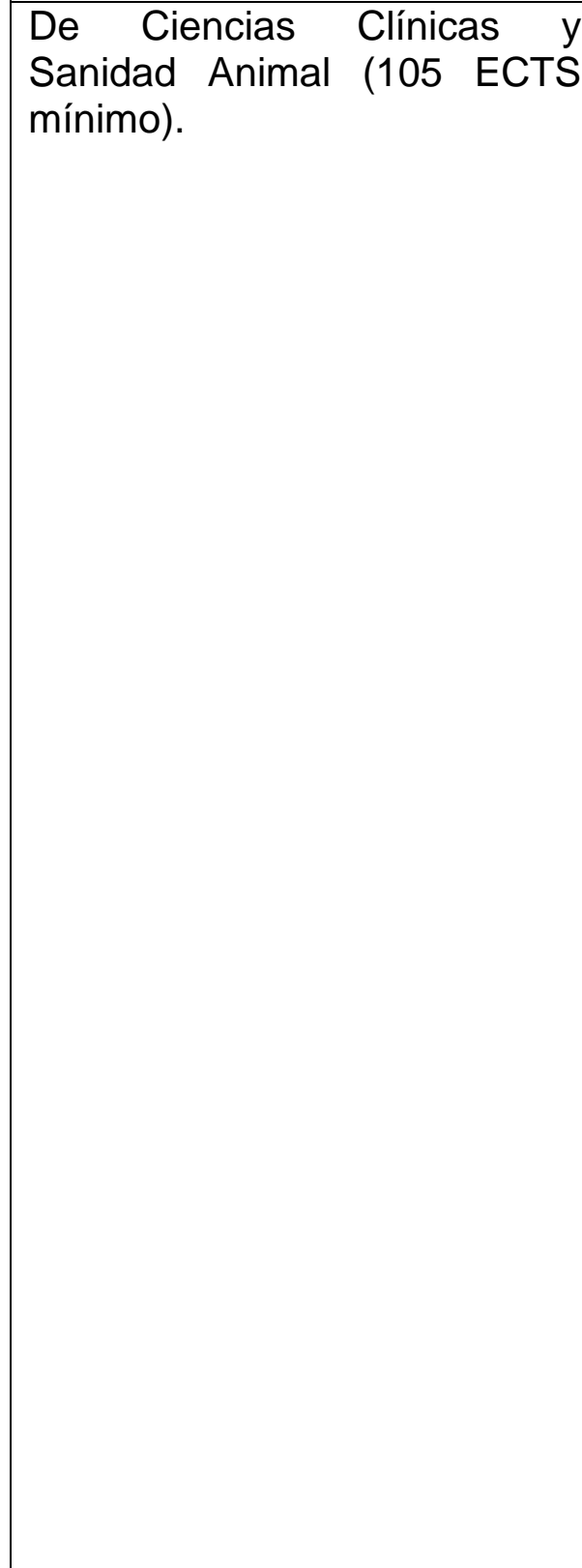 & $\begin{array}{l}\text { Métodos y procedimientos de exploración } \\
\text { clínica, técnicas diagnósticas } \\
\text { complementarias y su interpretación. } \\
\text { Diagnóstico por imagen y radiobiología. } \\
\text { Necropsia. Reconocimiento y diagnóstico de } \\
\text { los distintos tipos de lesiones y su asociación } \\
\text { con los procesos patológicos. Bases } \\
\text { farmacológicas generales y estudio de los } \\
\text { distintos tipos de drogas. } \\
\text { Estudio clínico del individuo enfermo y de los } \\
\text { tratamientos médicos, quirúrgicos o higiénico- } \\
\text { dietéticos que requiera, así como de las } \\
\text { enfermedades esporádicas que afecten a } \\
\text { colectivos. } \\
\text { Diagnóstico. Técnicas quirúrgicas utilizadas } \\
\text { en veterinaria. Anestesia y reanimación } \\
\text { animal. Reproducción, parto y puerperio: } \\
\text { Cuidados y enfermedades. Reproducción } \\
\text { asistida. Farmacoterapia. Identificación y } \\
\text { estudio de los tóxicos naturales y de síntesis. } \\
\text { Toxicología animal y medioambiental. } \\
\text { Ictiopatología. y mantenimiento de las } \\
\text { Transmisión y y métodos de estudio de las } \\
\text { enfermedades y métodiones. } \\
\text { enfermedades en las poblaciones. } \\
\text { Enfermedades infecciosas y parasitarias de } \\
\text { interés veterinario incluyendo su diagnóstico } \\
\text { y lucha. Zoonosis y Salud Pública. Promoción } \\
\text { de la salud en los colectivos animales, } \\
\text { incluidos los animales silvestres, con el fin de } \\
\text { obtener el máximo rendimiento económico de } \\
\text { una forma social, ética y sanitariamente } \\
\text { aceptables. } \\
\text { Mediadas técnicas y reglamentos para la }\end{array}$ \\
\hline
\end{tabular}




\begin{tabular}{|c|c|}
\hline & $\begin{array}{l}\text { prevención, control y erradicación de las } \\
\text { enfermedades animales. }\end{array}$ \\
\hline $\begin{array}{l}\text { De Producción Animal (30 } \\
\text { ECTS mínimo) }\end{array}$ & $\begin{array}{l}\text { Bases de la producción animal: Sistemas } \\
\text { tradicionales y actuales. Materias primas para } \\
\text { la alimentación animal: Características, } \\
\text { producción y conservación. Bases de la } \\
\text { nutrición animal, formulación de raciones y } \\
\text { fabricación de piensos. Aplicaciones } \\
\text { genéticas a programas de mejora y salud. } \\
\text { Estrategias y procedimientos reproductivos } \\
\text { aplicados a la producción. Fundamentos de } \\
\text { instalaciones ganaderas e higiene ambiental. } \\
\text { Economía del proceso productivo y } \\
\text { comercialización. Desarrollo sostenible. } \\
\text { La acuicultura. }\end{array}$ \\
\hline $\begin{array}{l}\text { De Higiene, Tecnología y } \\
\text { Seguridad Alimentaria (22 } \\
\text { ECTS mínimo) }\end{array}$ & $\begin{array}{l}\text { Componentes y características de los } \\
\text { alimentos. Procesos tecnológicos de } \\
\text { obtención, conservación y transformación de } \\
\text { los alimentos. Cambios, alteraciones y } \\
\text { adulteraciones que pueden sufrir. Criterios } \\
\text { sanitarios y bases legales de la inspección. } \\
\text { Inspección veterinaria ante y post mórtem. } \\
\text { Inspección de establecimientos y productos. } \\
\text { Buenas prácticas higiénicas, análisis de } \\
\text { peligros y puntos de control críticos. Control } \\
\text { de manipulación y tratamientos. Seguridad } \\
\text { Alimentaria y Salud Pública. } \\
\text { El análisis de riesgo alimentario: } \\
\text { Determinación, gestión y comunicación del } \\
\text { riesgo. La investigación de brotes de toxi- } \\
\text { infecciones alimentarias. Dinámica y } \\
\text { demografía de la infección y la intoxicación. } \\
\text { Epidemiología y diagnóstico. Sistema de } \\
\text { seguimiento y vigilancia. }\end{array}$ \\
\hline $\begin{array}{l}\text { Prácticas Tuteladas y } \\
\text { Trabajo } \\
\text { fin de Grado (30 } \\
\text { mínimo })\end{array}$ & $\begin{array}{l}\text { Prácticas preprofesionales, en forma de } \\
\text { rotatorio clínico independiente y con una } \\
\text { evaluación final de competencias, en } \\
\text { hospitales veterinarios universitarios, clínicas } \\
\text { ambulantes, granjas, plantas piloto, } \\
\text { departamentos con dispositivos destinados a } \\
\text { la docencia práctica en el grado de } \\
\text { veterinaria, así como estancias en } \\
\text { establecimientos veterinarios, así como } \\
\text { estancias en establecimientos veterinarios, } \\
\text { mataderos, empresas y organismos externos } \\
\text { del ámbito veterinario o afín. }\end{array}$ \\
\hline
\end{tabular}




\begin{tabular}{|l|l|}
\hline Trabajo fin de grado: Materia transversal \\
cuyo trabajo se realizará asociado a distintas \\
materias. \\
Conocimiento y aplicación práctica de los \\
principios y metodologías de la veterinaria, \\
así como la adquisición de las destrezas y \\
competencias descritas en los objetivos \\
generales del título.
\end{tabular}

Además hay que añadir la reglamentación propia del Ministerio y ANECA para estructurar los estudios, en la que aparece como materia obligatoria el "Trabajo Fin de Grado", dictando que los módulos básicos hay que desarrollarlos en los primeros cursos y que no puede haber asignaturas surgidas de competencias situadas en módulos diferentes.

De esta manera, las competencias que darían lugar a "Deontología, Medicina Legal y Legislación Veterinaria":

1. Parte de ellas se encuentran en el módulo Básico, lo que condena a la asignatura que surja de ellas a situarse en los primeros 2 cursos.

2. Las competencias asociadas a Medicina Legal no aparecen reflejadas en el módulo básico, por lo que hay que deducir que están reflejadas en el módulo de Ciencias Clínicas y Sanidad Animal.

Este segundo punto se puede comprobar en el proyecto VERIFICADO del Grado en Veterinaria (http://www.um.es/documents/14554/695331/MemoriaGradoVeterinaria\%28Mo dif-buena+2012.pdf/c81956e0-be3e-4c22-91e1-bf9ee84cbbbd), en la ficha de la materia (en el proyecto verificado los módulos se subdividen en materias) "Identificación, Bienestar Animal y Ética Profesional", de la que resultaría, entre otras, la asignatura "Deontología, Medicina Legal y Legislación Veterinaria". En esa ficha (página 58), en el apartado competencias específicas de la materia, las que surgen directamente de la Orden $\mathrm{ECl} 333 / 2008$, aparecen:

Específicas de la materia

- Características etnológicas y productivas, con especial referencia al manejo.

- Bases del comportamiento animal y del proceso de domesticación.

- Bienestar y protección animal.

- Bioética.

- Principios éticos de la profesión veterinaria.

- Normativa y reglamentación veterinaria.

Llama la atención que la directiva europea incluye por ejemplo la toxicología como ciencia básica, quedando finalmente incluida en ciencias clínicas y sanidad animal, permitiéndole estar, como dicta la lógica, en últimos cursos del título. Sin embargo esto no se realizó con deontología. 
Por otro lado la medicina legal y legislación veterinaria aparecen en la directiva europea en ciencias clínicas, lo cual le permitiría estar posicionada en últimos cursos del título, sin embargo queda finalmente diluida la medicina legal estando parte de sus contenidos en ciencias clínicas y sanidad animal, mientras que se relega la legislación veterinaria a la formación básica común. Es decir, que se "degrada" la legislación veterinaria a primeros cursos, algo totalmente carente de todo sentido académico.

Todo esto debe ser contextualizado, contemplando el hecho de que desde que se implantaron los estudios de veterinaria en España, la asignatura "Deontología, Veterinaria Legal y Legislación Veterinaria" a estado presente en con todos los planes de estudio, estando posicionada como dicta el sentido común en los últimos cursos del título, por la especificidad y por el carácter multidisciplinar que la conforman, precisando de conocimientos y competencias previamente asumidas por el estudiante en asignaturas de cursos anteriores, como se sigue haciendo por ejemplo en Medicina.

Todo ello lleva a plantearse bajo que criterios se permite por parte de las autoridades académicas implicadas, que por normativa ministerial se genere una sinrazón donde el principal perjudicado ha sido y es el estudiante de veterinaria, amén de un profesorado que asiste estupefacto al reto de explicar contenidos a un alumnado novel, que no dispone de los conocimientos mínimos para afrontar dicha asignatura. Es necesario señalar que un título con muchas semejanzas como es Medicina (carrera profesionalizante así como profesión regulada, que el Gobierno también normativiza) no se ha permitido semejante atropello.

Nos encontramos pues ante la lamentable situación, de cómo la interpretación de la norma y su aplicación de manera falta de criterio y sentido común, posibilita una situación dantesca y carente del más mínimo sentido docente, académico y universitario.

\section{BIBLIOGRAFÍA}

Directiva Europea 36/2005 del Parlamento Europeo y del Consejo, de 7 de septiembre de 2005, relativa al reconocimiento de cualificaciones profesionales, pág. 224. Disponible en: http://www.unizar.es/eees/doc/directiva200536ec.pdf. Última visita 13-11-2016.

Directiva Europea 55/2013 del Parlamento Europeo y del Consejo, de 20 de noviembre de 2013, por la que se modifica la Directiva 2005/36/CE relativa al reconocimiento de cualificaciones profesionales y el Reglamento (UE) no 1024/2012 relativo a la cooperación administrativa a través del Sistema de Información del Mercado Interior ("Reglamento IMI"). Disponible en: 
https://www.boe.es/diario boe/txt.php?id=DOUE-L-2013-82984. Última visita 13-11-2016.

Libro Blanco Título de Grado en Veterinaria. ANECA. 2005. Disponible en: http://www.aneca.es/var/media/150400/libroblanco jun05 veterinaria.pdf. Última visita 13-11-2016.

Orden ECI 333/2008 por la que se establecen los requisitos para la verificación de los títulos universitarios oficiales que habiliten para el ejercicio de la profesión de Veterinario. BOE n. 40 de 15 de febrero, pp: 8355-8357. Disponible en: http://www.boe.es/boe/dias/2008/02/15/pdfs/A08355-08357.pdf. Última visita 13-11-2016.

Proyecto VERIFICADO del Título de Grado en Veterinaria, pp. 58-60. Disponible en: http://www.um.es/documents/14554/695331/MemoriaGradoVeterinaria\%28Mod if-buena+2012.pdf/c81956e0-be3e-4c22-91e1-bf9ee84cbbbd. Última visita 1311-2016.

Real Decreto 1837/2008 por el que se incorporan al ordenamiento jurídico español la Directiva 2005/36/CE, del Parlamento Europeo y del Consejo, de 7 de septiembre de 2005, y la Directiva 2006/100/CE, del Consejo, de 20 de noviembre de 2006, relativas al reconocimiento de cualificaciones profesionales, así como a determinados aspectos del ejercicio de la profesión de abogado. BOE núm. 280 de 20 de noviembre, pp: 46185-46320. Disponible en: https://boe.es/diario boe/txt.php?id=BOE-A-2008-18702. Última visita 1311-2016. 